\title{
Android App Controlled Bluetooth Robot
}

\author{
Surbhi Verma \\ Student \\ Indore, M.P \\ India
}

\begin{abstract}
Now-a-days the world is optimizing and is becoming more precise by switching from the world of personal computers to laptops to android phones. Human is moving and is accepting compact technologies so that, the gap between personages and the machines is being reduced to ease the standard of living. The purpose of this project is to design and implement a compound robot. The compound robot will be able to move in four directions (left, right, forward, backward) and will detect the distance of the obstacle from the robot on the android app. The main intent of this project is to design and bring about a robot prototype by using Arduino Uno, Motor Driver L293D, HC05- Bluetooth module and to procure the goal of this project, to gain knowledge about Ultrasonic sensor HCSR-04, reconcilable software and controlled motor circuit need to be determined. The robot will have several characteristics like continuous display of distance from the obstacle on the app, easy handling of a robot with the help of an app rather than any remote controller.
\end{abstract}

\section{Keywords}

Smart phones, Bluetooth, Android OS, Robot, Motor.

\section{INTRODUCTION}

Smart phones are becoming a basic need in day to day life with massive storage capacities, fortified processors, ample divertissement functions and vast communicating methodologies. Bluetooth is mainly used for exchanging data between different devices be it two smart phones or be it a robot and a smart phone. It mainly performs data transmission and even improve the characteristics of the smart phone, it was developed by telecom vendor Ericsson in 1994, shows its merits by incorporation with smart phones. It has changed the medium of how people uses digital devices at home or offices and has brought wireless devices in existence. The basic element of a Bluetooth is piconet, which is a collection several slave devices operating together with one master. Maximum of seven slaves can share a common master through a same link. Even several piconet can link together and form scatternet. It is useful in home environments, looking at its range or normal working area be 8 meters. Bluetooth has gradually increased users to prosecute smart phones, which have gingerly turned into a multipurpose portable device and are accessible to people for their quotidian use.

Present day, android is widely accepted as an open source platform. Android consist of a complete package involving an operating system, middleware layer and core applications. A Smartphone is a cell phone built on a mobile computing platform, which has big number of boosted connectivity and computing ability than what a feature phone has.

In this paper, we are overcoming the problem of traditional robots, which are usually handled with any remote controller. Reducing the remote work we are making the robot move by just a click on the cell phone with android operating system.
The basic problem was a remote controller and batteries for the remote, which will be replaced by an android app. And further we are minimizing the power required to transfer any object from one place to another which can easily be transferred by the robot. Even the robot will be able to avoid crashing with other hardware cause of a sensor build inside the system.

This article is structured as follows: Section 2 consist of the purpose behind writing this paper and making the corresponding project, Section 3 present the background detail about the project, Section 4 include the design and implementation of the project, Section 5 resides the conclusion laid from the project and lastly Section 6 falls light on the future enhancements which can be made to the project.

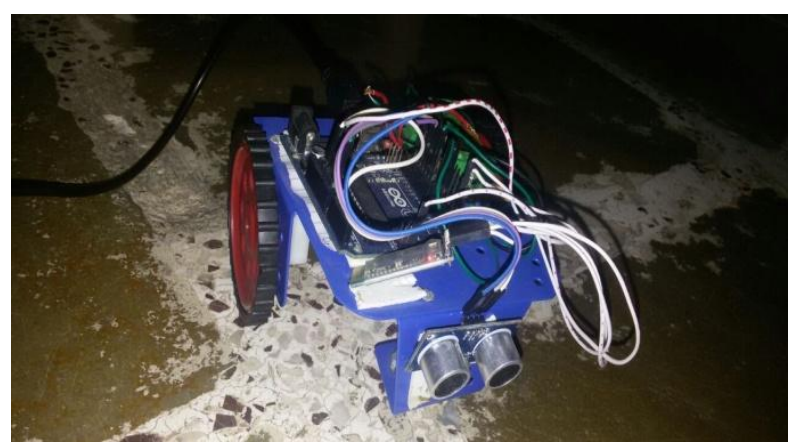

Fig 1: Bluetooth Robot

\section{PURPOSE}

The purpose behind this research is to design a robot for the transfer of data from one end to other in firms to make the information movement easier by just a tap on phone, with the help of an android application on one's cell phone. The android app will help the robot to move in different direction and transfer the material intended, in fact it will help the robot to not collide with any paraphernalia, as the distance of the obstacle will continuously get displayed on the screen of the app. By just a Bluetooth connectivity an android app can handle the movements of a robot and can move it in left, right, forward and backward directions.

\section{BACKGROUND}

\subsection{Motor Driver L293D}

L293D is a motor driver IC which allows the motor driver to move in any direction. With the help of motor driver, two DC motor can be attached on a single IC and both of them can be moved in either directions. L293D is a 16 pin IC which can control a set of direct current motors, Dual H-bridge Motor Driver integrated circuit(IC).This driver drives small as well as quite big motors, and it works on the concept of H-bridge circuit which allows the voltage to be flown in any direction. $\mathrm{H}$-bridge is ideal for driving a DC motor as the voltage needs to change the direction of the motor to make it move it in either clockwise or anti-clockwise direction. L293D pin 
diagram consist 4 input pins, 2, 7 are the left pins and 15, 10 are the right pins these pins regulate the rotation of the motor in either left side or right side. Inputs are given as Logic 0 (low) and Logic 1 (high), for rotating the motor the low and high signals are provided. It has Vcc pin where voltage required for internal operation is specified maximum of $5 \mathrm{~V}$ supply can be provided. Vss or ground pin is there where we can apply voltage for driving the motor, maximum of $36 \mathrm{~V}$ supply can be applied. Maximum of $600 \mathrm{~mA}$ current per channel can flow in the circuit.

\subsection{Motor}

Direct current motor is an electric motor which is capable of handling mechanical movements by converting conventional energy. DC motor takes electrical energy and produces mechanical energy. Dc motors are usually referred to as power devices, which are specifically used in auto mobiles, food blenders and so in robots. It is an electrical machine convertor which converts DC electric power to mechanical power and basically rely on the forces composed by magnetic field. It have either electro mechanical or electronics as internal mechanism to periodically change the direction of the current flow, mostly produces rotatory motion while some produce force directly and motion in the straight line.

\subsection{Arduino Uno}

UNO is a microcontroller based on ATmega328P. It has 14 digital input-output pins; 6 pins are for PWM outputs, 6 pins act as analog input pins. $16 \mathrm{MHz}$ crystal USB connector power jack is attached, consist of LCSP header and reset button. UNO contains everything needed to support any normal microcontroller $(\mu \mathrm{C})$. In UNO connections can be established by connecting Arduino to personal computer with a USB cable, power with AC to DC adapter can be provided or battery to get started. Arduino is a firm which design hardware, $\mu \mathrm{C}$ based kits for building digital devices and interactive objects that can percept and control physical devices. It establish a serial communication interface for loading programs from PC through USB.

\subsection{Arduino IDE}

IDE is an integrated development environment based on programming language named as Processing, it also support C and $\mathrm{C}++$. It basically is a cross-platform application written in JAVA. IDE is basically for software programming of any hardware board or IC. This code editor have following features: Syntax highlighting, brace matching, automatic indentation, one-click mechanism for loading and compiling of the programs on the Arduino board. In IDE program written is known as Sketch. $\mathrm{C} / \mathrm{C}++$ sketch consist of two functions which are compiled and amalgamate with a program stubmain().

- $\quad$ setup(): this function runs at the beginning or start of the program and even initialize the settings.

- $\quad \operatorname{Loop}()$ : this function is called repeatedly until the board power is cut-off.

\subsection{HC-05 Bluetooth Module}

This is used for establishing Bluetooth connectivity, it is easy to use Bluetooth SPP (special pot protocol) module. It setup a transparent wireless serial connection. $\mathrm{HC}-05$ is a fully qualified Bluetooth supporting V2.0+EDR(enhanced data rate), $3 \mathrm{Mbps}$ modulation, $2.4 \mathrm{GHz}$ baseband and use CSR Bluecore 04- External single chip Bluetooth system. Its footprint is very small limiting up to $12.7 \mathrm{~mm} \mathrm{X} 27 \mathrm{~mm}$, its default baud rate is 38400 . It underpin master and slave concept, and if the master and slave are paired then red and blue LEDs on the module blinks at 1 time per 2 seconds in interval and if disconnected blue LED blinks for 2 times per second. Its auto pairing pin code is " 0000 " as default and it automatically reconnect in $30 \mathrm{~min}$ when disconnected because of exciding the range of connection.

\subsection{Ultrasonic Sensor HC-sr04}

This sensor is attached to detect the distance of the obstacle from the robot. It uses sonar to govern distance of an object. It inaugurate non-contact range detection, and provide stable reading in an easy to use package. Its range varies from $2 \mathrm{~cm}$ to $400 \mathrm{~cm}$ or 1" to 13 feet. Sensor is not affected by sunlight or black material but it is difficult to detect the distance from any soft material like cloth. It is a combination of both ultrasonic transmitter and receiver module. Its output is greatly perturbed by Echo signal, so the output never goes Low if Echo is not received. Even timeout parameters are needed to alter the output according to the user aspirations. Its resolution is $0.3 \mathrm{~cm}$ and trigger input pulse width is $10 \mu \mathrm{S}$.

\subsection{Android Platform}

Android is a mobile operating system developed by Google based on Linus Kernel and is mostly wield with touch screen smart phones .Android platform is reliable for building an android application, android is a common choice of people while they buy any cell phone. Android provides easy accessibility and understandability of different aspects for daily use. They are widely known as mobile computers and are expanding the sale of smartphones worldwide. They are widely accepted as they provide open architecture, platform independent and enormous capabilities. It is built from JAVA programming language and as android uses JAVA, android API provide easy access for hardware components. Android may use USB, Wi-Fi and Bluetooth for connecting with the robot.

Android Studio: For building an android app android studio is looked for, android studio is an Integrated Development Environment (IDE) for android app development. The first stable build was released in December 2014, starting from version 1.0, based on JetBrains IntelliJ IDEA software, Android Studio is designed specifically for Android app development. It can be downloaded at Windows, Mac and Linux smoothly, and replaced Eclipse Android Development Tools (ADT) which is Google's primary IDE for indigene application development. It has Gradle based build support, Lint tools to catch performance and version compatibility. Android studio is a well-to-do layout editor which allows users to drag-and-drop UI components, and even provide user with option to preview layouts on multiple screens.

\section{DESIGN AND IMPLEMENTATION}

The robot is made up of an Arduino board, motor driver, 2 DC motors, Bluetooth module HC-05 \& Ultrasonic sensor. Firstly the data from the android app is sent as an input to the Bluetooth module which further gives it to the Arduino Uno, Uno is a controller which controls the signals and performs the assigned functions it understands which signals have to be forwarded to the motor driver so that it moves in particular order. Like in if the user have tapped the left button on the app the Arduino will send the signal to the motor driver to activate the left pins and accordingly move the motors so that the wheels can follow the direction.

As shown in the block diagram, User give directions through the app to the microcontroller with the help of Bluetooth module, then Arduino handles the motor driver which further 
supports the dc motors and enable the high signal at specific motor pins. The motor driver has several pins and those pins are for power supply, ground, and each dc motor have its own respective pins which when gets a high signal activates the dc motor, like pins 5,6 for the left motor and 9,10 for the right motor. Now the distance of the obstacle from the robot is calculated by the ultrasonic sensor which in turn gives a serial output on the app screen showing the distance.

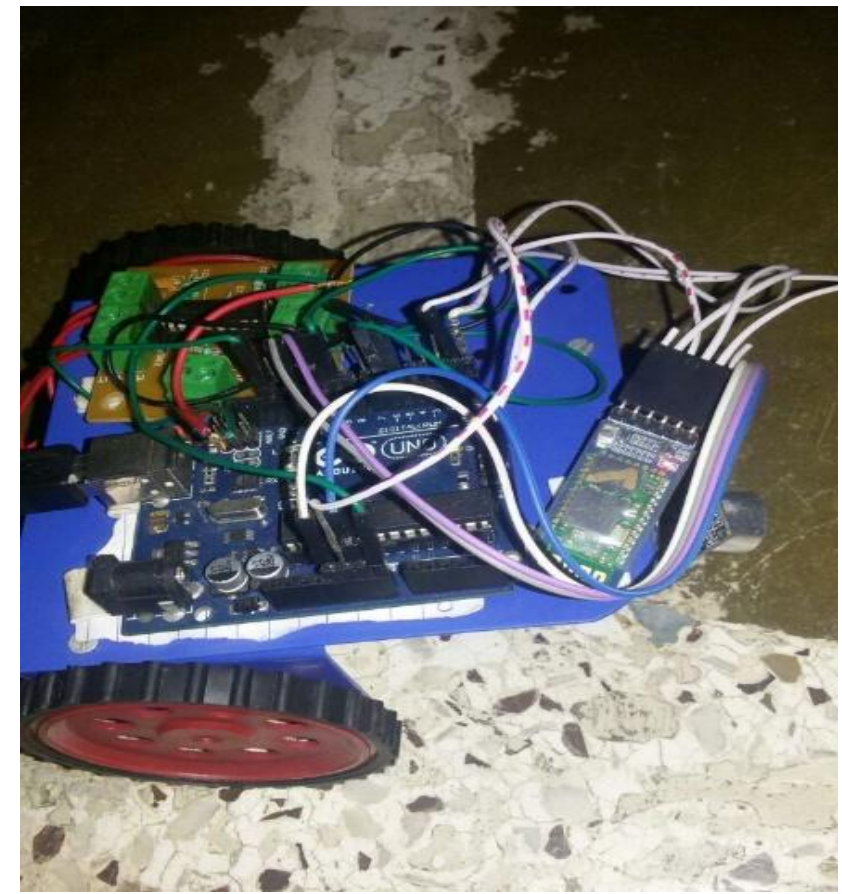

Fig 2: Android Controlled Bluetooth Robot

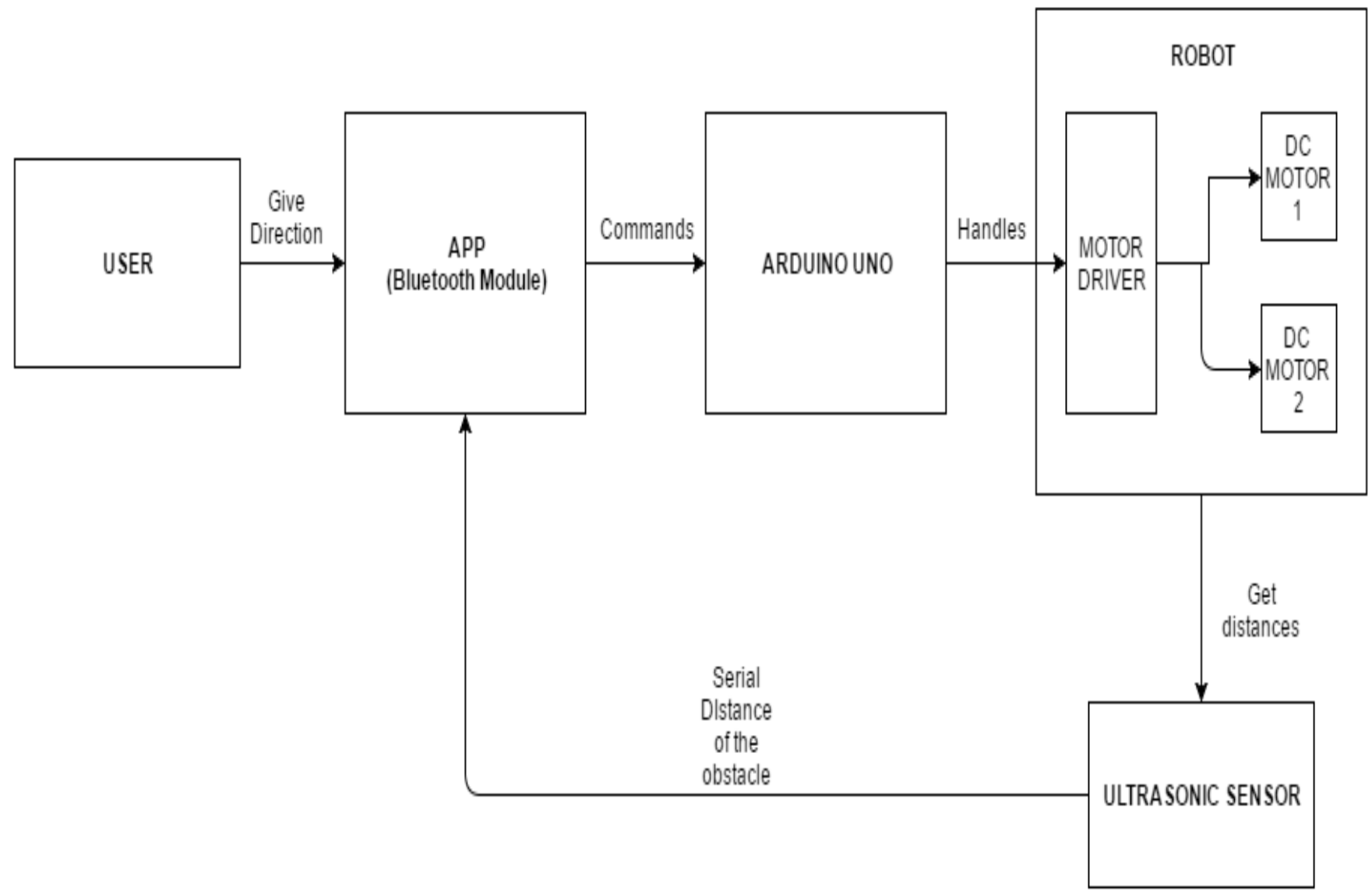

Fig 3: Block Diagram

\subsection{Controller Code}

The Arduino Uno board works according to the code burnt on it, code is burnt on the board with the help of a software.
- In code we specify maximum and minimum range of the sensor, input is taken from the Echo pin while output is 
sent to Trigger pin which is tied to the ping sensor or echo pin.

- Now with digitalWrite(pin, signal); command we give signals to the pin mentioned. For e.g.: digitalWrite $(5, \mathrm{HIGH})$; will produce a high signal on pin number 5 .

- A series of "IF" conditions is given for different directions: if(input $==$ "'f'); this will put a case when the $\mathrm{f}$ key is pressed the code will execute this if statement.

The high low signal works like if the high signal is given it moves the wheel in front and if low then backwards. Pins 5 of left motor \& 10 of right motor are responsible for moving in forward while pins 6 of left motor \& 9 of right motor are responsible for moving backward.

- For moving forward both motors receive high signal on pins 5, 10 and low signal on pins 6, 9 .

- For moving backward both motors receive high signal on pins 6, 9 and low signal on pins 5, 10.

- For moving in the right direction we need left motor to move in forward direction \& right motor to move in backward direction, so we will enable high signal at pins $5 \& 9$ and low signal at pins $6 \& 10$.

- For moving in the left direction we need right motor to move in forward direction \& left motor to move in backward direction, so we will enable high signal at pins $6 \& 10$ and low signal at pins $5 \& 9$.

For stop we will give low signal to all the pins.

\subsection{Application Instructions}

The application is generated through android studio.

- Just after getting started, app asks to enable the Bluetooth unless and until the Bluetooth module HC-05 is not connected, the app will not be able to move the robot.

- Console page consist of 5 buttons named as LEFT, RIGHT, FORWARD, BACKWARD \& STOP. On pressing these buttons one can move the robot in the specified direction, for example on pressing Right the app will send an $\mathrm{R}$ output to the controller which will then process the signal and give the input to motor driver. While pressing the stop button the robot will stop its movements.

- There is a display field where the distance from the Ultrasonic sensor is continuously displayed in centimeters.

- Lastly, an OK button is provided so once the robot stops and there are no further directions to be given user can click the button which will disconnect paired Bluetooth module.

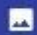

> $111: 17$

\section{ACTIONBOT}

BACKWARD

$\triangleleft$

$141 \mathrm{~cm}$

$139 \mathrm{~cm}$

$140 \mathrm{~cm}$

$141 \mathrm{~cm}$

$138 \mathrm{~cm}$

$142 \mathrm{~cm}$

$143 \mathrm{~cm}$

Fig 5: Continous Distance Display 


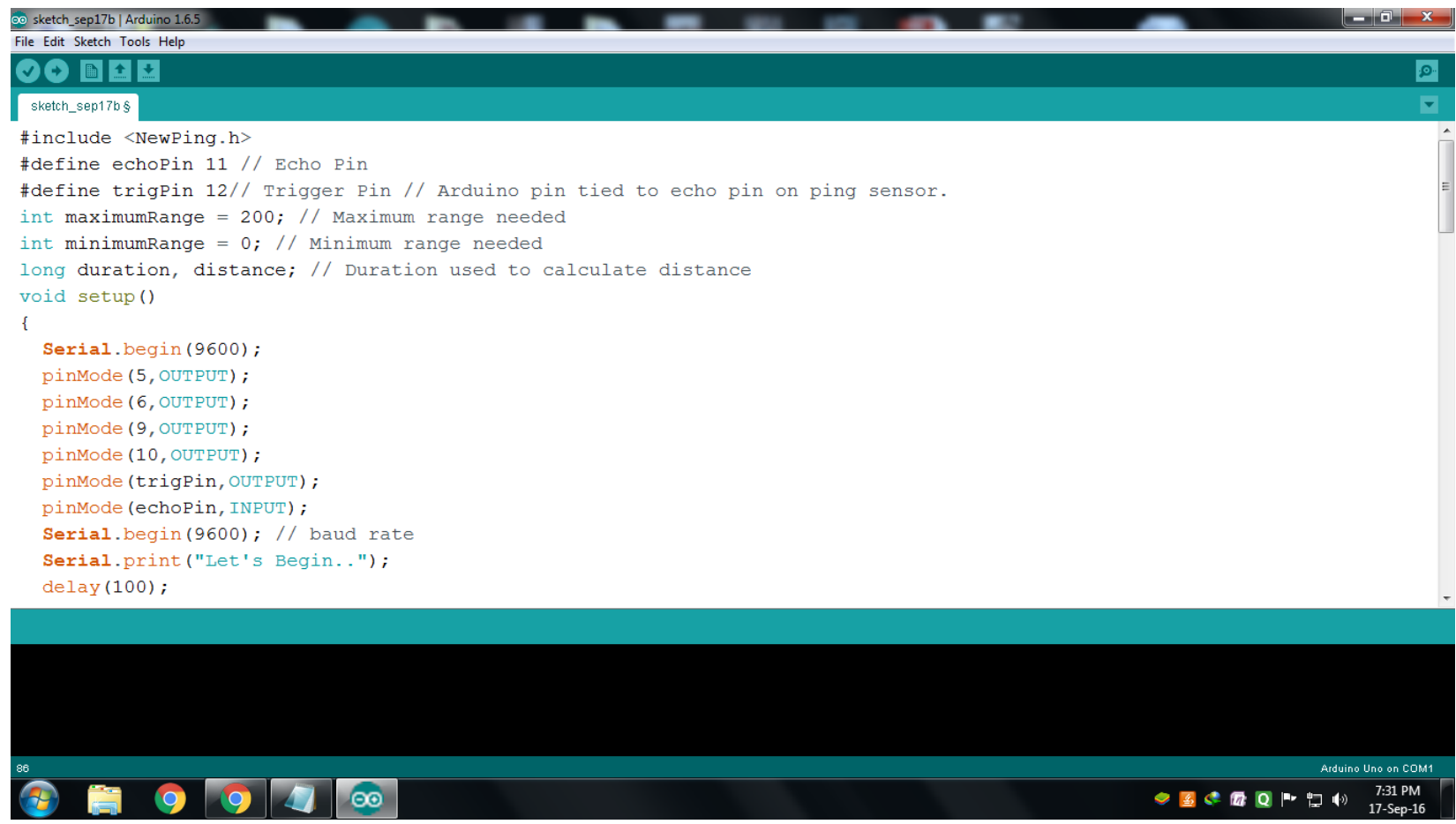

Fig 6: Arduino Code Implementation

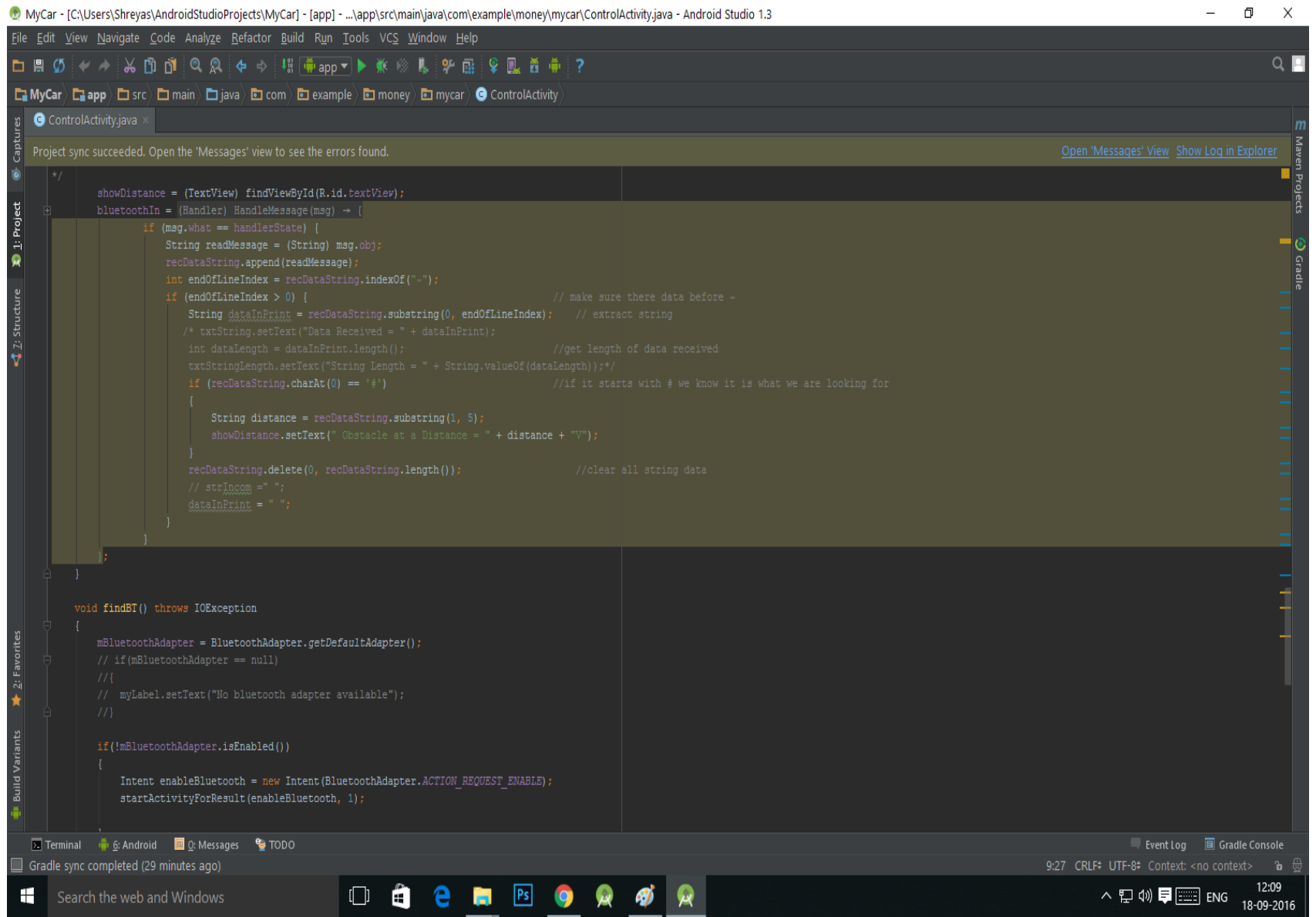

Fig 7: Android App Development

\section{CONCLUSION}

The purpose of writing this paper is to introduce a hardware system which can transfer small requirements from a room to another by just tapping on one's mobile screen. The hardware here introduced is a compound robot which is handled by an android application, the connectivity between the robot and the app is done by $\mathrm{HC}-05$ Bluetooth module, which provides 
5 meters range from its current position. Further to avoid crashing of robot with any other hardware is done by Ultrasonic sensor HC-sr04. Completion of this project will bring a new product to the world to increase speed and efficiency. The element which differentiate this research from other researches is introduction of the Ultrasonic sensor to detect the distance of the obstacle from the robot, by this one can change the movement of the robot before it wreck with any other thing. Thus it is concluded as by introduction of any such robot will enhance smart work and one can control their task remotely and wirelessly.

\section{FUTURE ENHANCEMENT}

Range of connectivity can be increased by using Wi-Fi. As of now we are using Bluetooth module which can be replaced by $\mathrm{Wi}-\mathrm{Fi}$ module. And can extend range by installing routers on short distances. Additional features can be added like to handle robot by voice commands, this is achieved by adding speech recognition module.

Movements and features of robot can be increased, by rotating it, and providing it diagonal movements. Camera can be attached and robot can be utilized in order to be used like a spy. More type of information and data can be transferred, head count in a room can be established, face recognition can be made, and by removing user id and password, login can be performed by checking finger prints.

\section{REFERENCES}

[1] Aiman Ansari, Yakub Ansari, Saquib Gadkari, Aarti Gokul "Android App Based Robot", Mumbai, India, 2015.

[2] Ritika Pahuja, Narender Kumar, "Android Mobile Phone Controlled Bluetooth Robot Using 8051 Microcontroller”, Bahal,India,2014.

[3] Wikipedia (Android app).

[4] Bharat Shresth Awasthi, Sabya Sanchi Pandey, Ashish Singh, Mrs.M.V. Patil "Robotic arm Wirelessly controlled by app", IJETR, June 2015.

[5] HC-06 bluetoothmodule, http://www. Lanwind.com/files/hc-06 en,pdf.

[6] Android tutorial, tutorialspoint.com.

[7] Google books, "Theory of Automatic Robot Assembly and Programming".

[8] Android tutorial, tutorialspoint.com.

[9] Arpit Sharma, Reetesh Verma, Saurabh gupta, Sukhdeep Kaur Bhatia,"Android Phone Controlled Robot Using Bluetooth”, Noida, India, 2014.

[10] Bluetooth Android Developers, https://developer.android.com/guide/topics/connectivity/ bluetooth.html.

[11] Wikipedia (Bluetooth).

[12] How does Bluetooth works, http://www.digitaltrends.com/mobile/how-doesbluetooth-work/. 